\title{
BMJ Open Measures of trunk muscle strength and their measurement properties: a protocol for a systematic review and narrative synthesis of clinical measures
}

\author{
Shouq Althobaiti, ${ }^{1}$ Alison Rushton (D) , ${ }^{1,2}$ Deborah Falla (D) , ${ }^{1}$ \\ Nicola R Heneghan (D) ${ }^{1}$
}

To cite: Althobaiti S, Rushton A, Falla D, et al. Measures of trunk muscle strength and their measurement properties: a protocol for a systematic review and narrative synthesis of clinical measures. BMJ Open 2021;11:e041499. doi:10.1136/ bmjopen-2020-041499

- Prepublication history and additional material for this paper are available online. To view these files, please visit the journal online (http://dx.doi. org/10.1136/bmjopen-2020041499).

Received 10 June 2020 Revised 26 November 2020 Accepted 18 December 2020

Check for updates

(C) Author(s) (or their employer(s)) 2021. Re-use permitted under CC BY-NC. No commercial re-use. See rights and permissions. Published by BMJ.

${ }^{1}$ Centre of Precision Rehabilitation for Spinal Pain (CPR Spine), School of Sport, Exercise and Rehabilitation Sciences, University of Birmingham, Birmingham, UK ${ }^{2}$ School of Physical Therapy, Elbourn College, Western University, London, Ontario, Canada

Correspondence to Dr Nicola R Heneghan; n.heneghan@bham.ac.uk

\section{ABSTRACT}

Introduction Spinal musculoskeletal conditions, including low back and neck pain, are leading causes of disability globally. The trunk muscles, which comprise muscles in the thoracic and lumbar regions, are essential for functional activities, necessitating mobility, motor control and strength. To investigate the effectiveness of interventions directed at improving trunk muscle strength, it is essential to have valid, reliable and responsive performance-based outcome measures (PBOM). While isokinetic dynamometry is considered the gold-standard PBOM, the associated costs, size/weight and operational complexity of this equipment preclude its use in a clinical setting. There is, therefore, a need to evaluate the measurement properties of alternative accessible measures of trunk strength. This systematic review therefore aims to investigate the measurement properties of PBOM of trunk muscle strength measures appropriate for use in a clinical setting.

Methods and analysis This protocol has been designed using the Preferred Reporting Items for Systematic Reviews and Meta-Analyses Protocols. CINAHL, Web of Science, Pedro, MEDLINE, EMBASE, SPORTDiscuss will be searched systematically from the database start date up to 16 April 2020, along with reference checking and the grey literature searching. Eligibility criteria include studies investigating measurement properties of PROM for trunk muscle strength for use in a clinical setting in adults with and without spinal musculoskeletal complaints. Two independent reviewers will determine the eligibility of the studies through screening process of titles, abstract and the full text. Both reviewers will assess the risk of bias using COnsensus-based Standards for the selection of health status Measurement Instruments risk-of-bias tool and then extract the data from included studies. The overall quality of the included studies will be evaluated using the Grading of Recommendations Assessment, Development and Evaluation approach. A narrative synthesis will be carried out if meta-analysis is not applicable. Findings from this systematic review will aid clinicians and practitioners working in the field for example, sport, in using the most appropriate PBOM to measure trunk muscle strength. Ethics and dissemination No research ethics application is needed as there are no patient data in this study. The results of this study will be submitted to a peer reviewed journal and presented at conferences.

\section{Strengths and limitations of this study}

- This is the first systematic review that will evaluate measurement properties of performance-based outcome measures, which will inform the selection of most reliable, valid and responsive tool to asses trunk muscle strength in a clinical setting.

- This study will use the consensus-based standards for the selection of health measurement instrument checklist, which therefore will improve the selection of trunk strength outcome measures in research and clinical practice.

- The term trunk is an umbrella term to reflect all muscles in the thoracic and lumbar spine, which may limit applicability of findings to specific clinical complaints in a specific spinal region.

PROSPERO registration number CRD42020167464.

\section{INTRODUCTION}

Musculoskeletal (MSK) conditions are common, not only among elderly but among children, teenagers and adults'. 'Back pain, neck pain, osteoarthritis and rheumatoid arthritis are considered among the most disabling MSK conditions that affect both physical and psychological capacities of individuals. $^{2}$ MSK disorders are considered the second highest contributor to years lived with disability. ${ }^{3}$ Low back pain (LBP) is the singleleading cause of disability worldwide ${ }^{3}$ and neck pain ranked as the fourth highest cause of years lived with disability. ${ }^{4}$ Therefore, spinal pain remains the main contributor of global disability. ${ }^{1}$ Recent data suggest that the lifetime prevalence of spinal pain is $20 \%-70 \%$ for neck pain, ${ }^{4} 3.7 \%-77 \%$ for thoracic spine pain $^{5}$ and $58 \%-84 \%$ for LBP, ${ }^{6}$ which place great economic burden on health services globally. In the UK, for instance, the estimated cost of managing chronic LBP alone is around £1.8-£2.3 billion. $^{7}$ 
Muscles of the trunk which includes those with attachments to both the thoracic and lumbar spines, are central to providing mobility and stability of the spine during functional activities, including, gait and daily life activities. ${ }^{8}$ Research has identified that weak trunk muscles are associated with exaggerated spinal curves, ${ }^{9}{ }^{10}$ and are a risk factor for spinal disorders ${ }^{11} 12$ and risk of falling. ${ }^{13}$ Several studies have suggested that patients with spinal MSK conditions may benefit from trunk strength training as essential part of the rehabilitation programmes. ${ }^{14}$ As a result, performance-based outcome measures (PBOM) of trunk muscle strength are important to evaluate patient clinical progression ${ }^{15}$ and to determine the effectiveness of therapeutic rehabilitation programmes. ${ }^{16}$

To evaluate trunk muscle strength, manual muscle testing, ${ }^{17}$ hand-held dynamometer ${ }^{18}$ strain gauge tests, ${ }^{19}$ isostation ${ }^{20}$ and isokinetic test ${ }^{21}$ have been described as available methods. However, the PROM need to obtain good level of measurement properties to be clinically and scientifically useful to help guide clinical decision making and treatment monitoring. ${ }^{22}{ }^{23}$ A measurement property is the quality aspect of an instrument and due to variations in the terminology and definitions of these measurement properties, the Consensus-based Standards for the Selection of Health Measurement Instruments (COSMIN) initiative developed a consensus-based taxonomy of measurement properties. ${ }^{24}$ The aforementioned taxonomy covers the three main domain (reliability, validity and responsiveness). ${ }^{25}$

Several literature reviews have been published summarising and critically appraising the trunk muscle strength PBOM. ${ }^{26-31}$ However, few have assessed their measurement properties. Two reviews have evaluated the measurement properties of trunk muscle strength using isomachines (isokinetic and isostation) ${ }^{28}{ }^{29}$ Acceptable levels of reliability were reported for flexion and extension up to $120^{\circ} / \mathrm{s}$, with limited and conflicting evidence regarding the reliability of trunk lateral bending strength. ${ }^{29}$ However, conflicting evidence also exists regarding the validity of Iso-machines across both reviews. ${ }^{28} 29$

Other reviews have reported some measurement properties of trunk muscle strength measures in neurological conditions. ${ }^{3031}$ However, the reliability of the PBOM with one group does not necessarily generalise to another ${ }^{32}$ and the value of these measures for use in a spinal MSK population is questionable. ${ }^{33}$ Establishing measurement properties of any PBOM within a defined population is important to eliminate any potential bias and to have confidence in findings. ${ }^{25}$ To the best of the author's knowledge, no systematic review has been published targeting the psychometric properties of the trunk muscle strength PBOM for use in a clinical setting. Therefore, a systematic review is needed to comprehensively evaluate the psychometric properties of the different clinical trunk strength outcome measures in healthy participants and patients with spinal MSK complaints. This systematic review aims to assess the measurement properties (validity, reliability and responsiveness) of trunk muscle strength PBOM for use in a clinical setting.

\section{Aim}

To evaluate the measurement properties (validity, reliability and responsiveness) of the trunk muscle strength outcome measures appropriate for use in routine clinical practice.

\section{METHODS}

The systematic review protocol is designed using The Cochrane Handbook for Diagnostic Test Accuracy studies and the Centre for Reviews and Dissemination ${ }^{34}$ and reported in line with The Preferred Reporting Items for Systematic Reviews and Meta-Analyses (PRISMA) Protocols guidelines checklist, ${ }^{35}$ (see online supplemental file 1). TheCOSMIN group developed guidelines and practical tools for conducting studies on measurement properties or selecting the high-quality studies for systematic reviews on measurement properties. ${ }^{3637}$ Hence, COSMIN risk-of-bias checklist will be used to improve the selection of the available trunk strength outcome measures available.

\section{ELIGIBILITY CRITERIA}

\section{Inclusion criteria}

Eligibility criteria will include adult participants aged $\geq 18$ years who are either healthy or experiencing any spinal MSK condition. For the purpose of this review, spinal MSK conditions will be defined based on the International Classification of Diseases as any condition that affects the spine bones, joints, muscles and associated tissues such as ligaments and tendons. ${ }^{38}$ (eg, arthritis, neck pain, thoracic pain, osteoporosis, scoliosis, LBP, etc). Including healthy participants will help to identify the cut-off points in trunk strength measures between healthy individuals and those with spinal MSK complaints. We will include studies which have investigated any PBOM of trunk muscle strength performed in a clinical or field-based setting, including manual, functional and mechanical methods. Eligible studies must have evaluated one or more aspect of the main three domains of the COSMIN Taxonomy (validity, reliability and responsiveness) of measurement properties will be included.$^{25}$ More details on the three domains of the COSMIN Taxonomy for measurement properties can be found in online supplemental file 2. Studies must report on the evaluation of the measurement properties of PBOM of trunk muscle strength which can be done in a clinical or field-based setting.

\section{Exclusion criteria}

Any study written in a language other than English will be excluded. Studies reporting measurement properties of PBOM which involve expensive, technical equipment or computerised instruments namely; isokinetic and isostation machines ${ }^{27}$ and are not practical, ${ }^{159}{ }^{39}$ that is, relevant 
for clinicians working in home-based, community-based or field-based settings who need portable, easy to use devices ${ }^{40}$ will be excluded.

\section{Information sources}

A comprehensive search strategy will be developed using both medical subject headings and free text, relevant keywords identified during the scanning search. Following the Cochrane collaboration recommendations, multiple electronic databases and a subject-specific database will be searched systematically, to cover the broadest available literature. These include CINAHL and SPORTDiscuss via (EBSCO interface), MEDLINE and EMBASE through (Ovid interface), Web of Science and Pedro. The search was from the database start date up to April 16, 2020, with no restrictions to the publication time frame although only articles published in English was included in this review. Handsearching through checking reference lists and grey literature searching through the main sources such as British National bibliography for report literature and open Grey will be conducted as well.

\section{Search strategy}

The search strategy was developed in discussion with the supervisory team (NRH, AR and DF) and a specialist librarian. Initially, the search strategy will be developed in the MEDLINE database, and then it will be adapted for each database. Specific key terms will include terms related to psychometric properties e.g. reliability, validity and responsiveness as well as terms describing the population of interest will be used. Additional search filters designed by COSMIN for retrieving studies on measurement properties will be applied where appropriate. ${ }^{41}$ See example of search strategy in online supplemental file 3. Relevant studies will be identified and selected by two independent reviewers $\mathrm{SA}$ and $\mathrm{AA}$ with specialist training and knowledge in MSK rehabilitation and research methods.

\section{Study records}

\section{Data management}

Comprehensive search on the after mentioned databases will be carried out by the main author SA. All search results will be exported and stored on EndNote V. X9 (Clarivate analytics) software programme. This will allow any duplicates to be identified and subsequently removed as well as storing of abstract and full texts.

\section{Selection process}

Secondary to searching process, two reviewers SA and AA will independently screen titles and abstract based on preidentified eligibility criteria and will subcategorise the identified studies into include/exclude/unsure. ${ }^{42}$ The second step comprises retrieving and reading the full text of potentially relevant articles which will then be independently examined by each reviewer against the eligibility criteria. If further information needed, authors will be contacted via email. Agreement between both reviewers is required for the study to be included in the review.
Table 1 Summary of data to be extracted from included studies

\begin{tabular}{ll}
\hline Content & Data items \\
\hline $\begin{array}{l}\text { Bibliographic data } \\
\text { characteristics }\end{array}$ & $\begin{array}{l}\text { Authors, year of publication } \\
\text { Study design, sample size }\end{array}$ \\
$\begin{array}{l}\text { Setting } \\
\text { Participants } \\
\text { characteristics }\end{array}$ & $\begin{array}{l}\text { Age, gender, healthy or with spinal } \\
\text { musculoskeletal condition. }\end{array}$ \\
$\begin{array}{l}\text { Outcome measures } \\
\text { Trunk muscle strength measures. } \\
\text { Type of muscle contraction measured. } \\
\text { Measurement procedure: warm-up, } \\
\text { participants' position, fixation, examiner } \\
\text { position (if any), line of force (resistance), } \\
\text { cool down. }\end{array}$ \\
$\begin{array}{l}\text { Measurement properties (reliability, validity } \\
\text { and responsiveness), statistical methods } \\
\text { used and results. }\end{array}$ \\
\hline $\begin{array}{l}\text { Measurement } \\
\text { properties }\end{array}$
\end{tabular}

Therefore, agreement will be assess using Cohen's kappa (k) statistic disagreement will be resolved by consensus or by the decision of a third reviewer NRH. ${ }^{43}$ Information regarding the excluded studies and the reason for exclusion will be reported using PRISMA flow chart.

\section{Data collection process}

For each included study, a standard form will be used to extract the data. To ensure all the relevant information is captured, piloting the data collection form will be conducted. Both reviewers SA and AA will independently extract the data using the standardised form, the authors will be contacted for more clarification or if there is any missing data. In case of disagreement about extracted data between reviewers, discussion and/or involving a third reviewer (NRH) will be carried out until consensus reached.

\section{Data items}

Table 1 summarise the relevant data to be extracted from included studies.

\section{Risk of bias in individual studies}

The COSMIN Risk of Bias checklist for systematic reviews will be used to evaluate the risk of bias of included studies in this review. ${ }^{44}$ The COSMIN Risk of Bias Checklist considered to have adequate reliability as it is developed from the original COSMIN tool which show high percentage inter-rater agreement. ${ }^{45}$ The COSMIN Risk of Bias checklist includes standards for both design and the preferred statistical methods for each measurement property. The checklist covers nine different dimensions of reliability, validity and responsiveness. The COSMIN checklist was originally designed to evaluate patientreported outcome measures (PROM). However, the COSMIN group have recommended adaptation of the tool for use with other types of measures such as clinicianreported outcome measures or $\mathrm{PBOM}^{41}$ As with the 
study selection process, two raters SA and AA will independently score each outcome measure as either 'very good', 'adequate', 'doubtful' or 'inadequate' quality. ${ }^{44}$ Any disagreement between the reviewers will be resolved through discussion, if no consensus can be reached, a third reviewer will be consulted.

\section{Data synthesis}

Depending on the heterogeneity of included studies, either a meta-analysis or narrative synthesis will be conducted to synthesise the results which will follow the COSMIN guidelines for systematic reviews. ${ }^{44}$ The results from different studies on single measurement property will be quantitatively pooled in meta-analysis if sufficient number of studies share the same reference standard, designs, population and measure the same movement. To find the estimate of test-retest reliability, standard generic inverse variance random effects model will be implemented to calculate the weighted mean intraclass correlation coefficients and 95\% CIs. For construct validity, all correlations of PBOM with other PBOM that measure the same construct will be pooled. ${ }^{44}$ Following the assessment of scoping searches of the currently available literature, pooling of data might not be possible due to an anticipated lack of homogeneity. Hence, a narrative synthesis will be conducted in line with the narrative synthesis in systematic reviews recommendation. ${ }^{46}$ Synthesis will bring together evidence of measures of trunk strength, summary table will be generated to illustrates the pooled results per each measurement property per outcome measure per movement and rated against the updated criteria for good measurement properties as; sufficient $(+)$, insufficient $(-)$, inconsistent $( \pm)$ or indeterminate (?) ${ }^{44}$ Further analysis of the results will be presented in the discussion section in line with the quality of evidence.

\section{Meta-bias}

To eliminate any chance of publication bias, grey literature and conference papers will be searched.

\section{Confidence in cumulative evidence}

The overall quality of evidence regarding the measurement properties will then be assessed using a modified Grading of Recommendations Assessment, Development and Evaluation (GRADE) approach. As recommended by COSMIN guidelines for systematic review, a modified GRADE approach will be used to assess how the pooled results from included studies are trustworthy. ${ }^{36}$ The quality of evidence will be determined using four factors from the GRADE approach: risk of bias, inconsistency, imprecision and indirectness. Following the COSMIN recommendation, the fifth factor, that is, publication bias will not be used due to lack of registry data on measurement properties studies.

\section{Patient and public involvement}

The study question and systematic review protocol were informed following many years of working with patients and clinical experiences of managing patients with spinal dysfunction. The question was informed following discussions with patient and public involvement meeting at the Centre of Precision Rehabilitation for Spinal Pain and specifically in light our earlier research investigating measurement properties of PBOM for spinal mobility. The group consist individuals with different MSK and spinal complaints. Since no patient data is needed, patients will not be involved in data collection or analysis. However, the results of the study will be shared with this group and other public engagement events.

\section{Clinical implications of this study}

By accurately measuring the trunk muscles strength, diagnosis of dysfunction as well as improvement can be monitored. Also, with the current assessment methods available to measure trunk strength, finding a valid, reliable and responsive tool as well as cost-effective for clinical use is a priority. Noteworthy, with the vast range of different test procedures and positions used, this review will summarise data regarding the measurement properties of different assessment methods and highlight the method which is superior in terms of psychometric properties, cost-effectiveness and time-saving. In doing so, healthcare professionals will be aware of the valid method to use within the clinical setting to assess the effectiveness of interventions directed to improve muscular function. Using accurate and objective muscle strength measures will facilitate the monitoring of rehabilitation programme efficacy and effectiveness of targeted interventions to improve trunk muscle strength.

\section{ETHICS AND DISSEMINATION}

No patient data will be collected, hence no ethical approval is needed for this systematic review. The results of this review will help to inform current healthcare practice and research on the most valid, reliable and responsive tool for measuring trunk muscle strength. Results of this review will be submitted to be published in a peerreview journal and presented at relevant conferences.

Twitter Alison Rushton @abrushton, Deborah Falla @Deb_Falla and Nicola R Heneghan @HeneghanNicola

Contributors SA is a PhD student at the University of Birmingham. NRH, AR and DF are supervisors. NRH and AR have expertise in the field of outcome measurement methods. SA, NRH, ABR and DF contributed to the systematic review topic. SA drafted the protocol with guidance and feedback from NRH, AR and DF. NRH, AR and $\mathrm{DF}$ reviewed the manuscript and commented on the protocol. AA is a PhD student who will act as a second reviewer. All authors have approved and contributed to the final manuscript.

Funding The authors have not declared a specific grant for this research from any funding agency in the public, commercial or not-for-profit sectors.

Competing interests None declared.

Patient consent for publication Not required.

Provenance and peer review Not commissioned; externally peer reviewed.

Supplemental material This content has been supplied by the author(s). It has not been vetted by BMJ Publishing Group Limited (BMJ) and may not have been peer-reviewed. Any opinions or recommendations discussed are solely those of the author(s) and are not endorsed by BMJ. BMJ disclaims all liability and responsibility arising from any reliance placed on the content. Where the content 
includes any translated material, BMJ does not warrant the accuracy and reliability of the translations (including but not limited to local regulations, clinical guidelines, terminology, drug names and drug dosages), and is not responsible for any error and/or omissions arising from translation and adaptation or otherwise.

Open access This is an open access article distributed in accordance with the Creative Commons Attribution Non Commercial (CC BY-NC 4.0) license, which permits others to distribute, remix, adapt, build upon this work non-commercially, and license their derivative works on different terms, provided the original work is properly cited, appropriate credit is given, any changes made indicated, and the use is non-commercial. See: http://creativecommons.org/licenses/by-nc/4.0/.

\section{ORCID iDs}

Alison Rushton http://orcid.org/0000-0001-8114-7669

Deborah Falla http://orcid.org/0000-0003-1689-6190

Nicola R Heneghan http://orcid.org/0000-0001-7599-3674

\section{REFERENCES}

1 Briggs AM, Woolf AD, Dreinhöfer K, et al. Reducing the global burden of musculoskeletal conditions. Bull World Health Organ 2018;96:366.

2 Ingram M, Symmons DPM. The burden of musculoskeletal conditions. Medicine 2018;46:152-5.

3 GBD 2017 Disease and Injury Incidence and Prevalence Collaborators. Global, regional, and national incidence, prevalence, and years lived with disability for 354 diseases and injuries for 195 countries and territories, 1990-2017: a systematic analysis for the global burden of disease study 2017. Lancet 2018;392:1789-858.

4 Blanpied PR, Gross AR, Elliott JM, et al. Neck pain: revision 2017. J Orthop Sports Phys Ther 2017;47:A1-83.

5 Heneghan NR, Rushton A. Understanding why the thoracic region is the 'Cinderella' region of the spine. Man Ther 2016;21:274-6.

6 Bernstein IA, Malik Q, Carville S, et al. Low back pain and sciatica: summary of NICE guidance. BMJ 2017;356:i6748.

7 Hong J, Reed C, Novick D, et al. Costs associated with treatment of chronic low back pain: an analysis of the UK general practice research database. Spine 2013;38:75-82.

8 Kong YS, Cho YH, Park JW. Changes in the activities of the trunk muscles in different kinds of bridging exercises. J Phys Ther Sci 2013;25:1609-12.

9 Katzman WB, Wanek L, Shepherd JA, et al. Age-related hyperkyphosis: its causes, consequences, and management. $J$ Orthop Sports Phys Ther 2010;40:352-60.

10 Barczyk-Pawelec K, Piechura JR, Dziubek W, et al. Evaluation of isokinetic trunk muscle strength in adolescents with normal and abnormal postures. J Manipulative Physiol Ther 2015;38:484-92.

11 Biering-Sørensen F. Physical measurements as risk indicators for low-back trouble over a one-year period. Spine 1984;9:106-19.

12 Steele J, Bruce-Low S, Smith D. A reappraisal of the deconditioning hypothesis in low back pain: review of evidence from a triumvirate of research methods on specific lumbar extensor deconditioning. Curr Med Res Opin 2014;30:865-911.

13 Kato S, Murakami H, Demura S, et al. Abdominal trunk muscle weakness and its association with chronic low back pain and risk of falling in older women. BMC Musculoskelet Disord 2019;20:273.

14 Gordon R, Bloxham S. A systematic review of the effects of exercise and physical activity on non-specific chronic low back pain. Healthcare 2016;4. doi:10.3390/healthcare4020022. [Epub ahead of print: 25 Apr 2016]

15 El Mhandi L, Bethoux F. Isokinetic testing in patients with neuromuscular diseases: a focused review. Am J Phys Med Rehabil 2013:92:163-78.

16 Demoulin C, Grosdent S, Smeets R. Muscular performance assessment of trunk extensors: a critical appraisal of the literature: low back pain. IntechOpen, 2012

17 Petty NJ, Examination N. Neuromusculoskeletal examination and assessment, a Handbook for therapists with PAGEBURST access, 4 neuromusculoskeletal examination and assessment. Elsevier Health Sciences, 2011.

18 Durmus D, Akyol Y, Alayli G, et al. Effects of electrical stimulation program on trunk muscle strength, functional capacity, quality of life, and depression in the patients with low back pain: a randomized controlled trial. Rheumatol Int 2009:29:947-54.

19 da Silva RA, Arsenault AB, Gravel D, et al. Back muscle strength and fatigue in healthy and chronic low back pain subjects: a comparative study of 3 assessment protocols. Arch Phys Med Rehabil 2005;86:722-9.

20 Deutsch S. B-200 back evaluation system version 3.0. Pawtucket, RI: Occupational Orthopedic Center, 1989.
21 Newton M, Thow M, Somerville D, et al. Trunk strength testing with iso-machines. Part 2: experimental evaluation of the Cybex II back testing system in normal subjects and patients with chronic low back pain. Spine 1993;18:812-24.

22 Lexell JE, Downham DY. How to assess the reliability of measurements in rehabilitation. Am J Phys Med Rehabil 2005;84:719-23.

23 Roach KE. Measurement of health outcomes: reliability, validity and responsiveness. J Prosthet Orthot 2006;18:P8-12.

24 De Vet HC, Terwee CB, Mokkink LB. Measurement in medicine: a practical guide. Cambridge University Press, 2011.

25 Mokkink LB, Terwee CB, Patrick DL, et al. The COSMIN study reached international consensus on taxonomy, terminology, and definitions of measurement properties for health-related patientreported outcomes. J Clin Epidemiol 2010;63:737-45.

26 Beimborn DS, Morrissey MC. A review of the literature related to trunk muscle performance. Spine 1988;13:655-60.

27 Malliou P, Gioftsidou A, Beneka A, et al. Measurements and evaluations in low back pain patients. Scand J Med Sci Sports 2006:16:219-30.

28 Mueller S, Stoll J, Mueller J, et al. Validity of isokinetic trunk measurements with respect to healthy adults, athletes and low back pain patients. Isokinet Exerc Sci 2012;20:255-66.

29 Newton M, Waddell G. Trunk strength testing with iso-machines. Part 1: review of a decade of scientific evidence. Spine 1993;18:801-11.

30 Jørgensen M, Dalgas U, Wens I, et al. Muscle strength and power in persons with multiple sclerosis - A systematic review and metaanalysis. J Neurol Sci 2017;376:225-41.

31 Rabelo M, Nunes GS, da Costa Amante NM, et al. Reliability of muscle strength assessment in chronic post-stroke hemiparesis: a systematic review and meta-analysis. Top Stroke Rehabil 2016;23:26-35

32 Dijkers MPJM, Kropp GC, Esper RM, et al. Reporting on reliability and validity of outcome measures in medical rehabilitation research. Disabil Rehabil 2002;24:819-27.

33 Portney LG, Mary P. Foundations of clinical research: applications to practice. 2 edn, 2002: 598-98.

34 Deeks J, Wisniewski S, Davenport C. Guide to the contents of a Cochrane Diagnostic Test Accuracy Protocol. In: Deeks J, Bossuyt P. Gatsonis C, eds. Cochrane Handbook for systematic reviews of diagnostic test accuracy version 100. The Cochrane Collaboration, 2013.

35 Moher D, Shamseer L, Clarke M, et al. Preferred reporting items for systematic review and meta-analysis protocols (PRISMA-P) 2015 statement. Syst Rev 2015;4:1.

36 Mokkink LB, Prinsen CA, Patrick DL. COSMIN methodology for systematic reviews of Patient-Reported outcome measures (PROMs) user manual. 78. Netherlands, 2018.

37 Mokkink LB, Terwee CB, Patrick DL, et al. The COSMIN checklist for assessing the methodological quality of studies on measurement properties of health status measurement instruments: an international Delphi study. Qual Life Res 2010;19:539-49.

38 Campbell LC. Musculoskeletal disorders: addressing disparities in prevalence, severity, and treatment. N C Med J 2017;78:315-7.

39 Stark T, Walker B, Phillips JK, et al. Hand-held dynamometry correlation with the gold standard isokinetic dynamometry: a systematic review. Pm R 2011;3:472-9.

40 Trudelle-Jackson E, Jackson AW, Frankowski CM, et al. Interdevice reliability and validity assessment of the Nicholas hand-held dynamometer. J Orthop Sports Phys Ther 1994;20:302-6.

41 Prinsen CAC, Mokkink LB, Bouter LM, et al. COSMIN guideline for systematic reviews of patient-reported outcome measures. Qual Life Res 2018;27:1147-57.

42 McKenzie JE BS, Ryan RE, Thomson HJ. Defining the criteria for including studies and how they will be grouped for the synthesis. In: Higgins JPT TJ, Chandler J, Cumpston M, eds. Cochrane Handbook for systematic reviews of interventions version 60, 2019.

43 Catalin Tufanaru ZM, Aromataris E, Campbell J. Systematic reviews of effectiveness. In: Aromataris EMZ, ed. JBI reviewer's manual, 2017.

44 Mokkink LB, de Vet HCW, Prinsen CAC, et al. COSMIN risk of bias checklist for systematic reviews of patient-reported outcome measures. Qual Life Res 2018;27:1171-9.

45 Mokkink LB, Terwee CB, Gibbons E, et al. Inter-rater agreement and reliability of the COSMIN (consensus-based standards for the selection of health status measurement instruments) checklist. BMC Med Res Methodol 2010;10:82.

46 Popay J, Roberts H, Sowden A. Guidance on the conduct of narrative synthesis in systematic reviews: a product from the ESRC methods programme 2006;1:b92. 